\title{
Splenic sarcoidosis: A case report and review of the imaging findings of multiple incidental splenic lesions as the initial presentation of sarcoidosis
}

Earl L Raber MD FRCPC ${ }^{1}$, J Haba MD ${ }^{1}$, Paul Beck PhD MD FRCPC ${ }^{2}$

A case involving a 37-year-old woman with a new diagnosis of celiac disease who underwent ultrasound for abdominal pain that showed multiple splenic lesions is presented. Because metastatic disease was raised as a probable diagnostic consideration, an enhanced computed tomography (CT) scan of the abdomen was performed that confirmed the lesions but was otherwise indeterminate (Figure 1). The splenic lesions were further evaluated with magnetic resonance imaging (MRI) and found to have features most characteristic of splenic sarcoid (Figure 2).

Interstitial lung changes were noted at the lung bases on chest radiography (Figure 3A) and abdominal CT. Subsequent high-resolution CT revealed the lung changes to be diffuse with a classic central bronchovascular reticular-nodular interstitial pattern most suggestive of sarcoidosis (Figure 3B); tissue diagnosis of sarcoidosis was confirmed at bronchoscopy.

Because the patient was relatively asymptomatic, she was managed conservatively. The splenic lesions and the pulmonary changes were followed with imaging for more than one year. Both demonstrated improvement, with the splenic lesions significantly decreasing in size and number (Figure 4).

\section{DISCUSSION}

Sarcoidosis is a multisystem disease of unknown etiology characterized by proliferation of noncaseating granulomas. One of the favoured theories is that sarcoidosis is the end result of immune responses to various ubiquitous environmental triggers in patients with a genetic predisposition. Its presentation varies widely, it affects individuals of all racial and ethnic groups and it can occur at any age; however, its peak incidence occurs between 20 and 39 years of age with a female predominance (1).

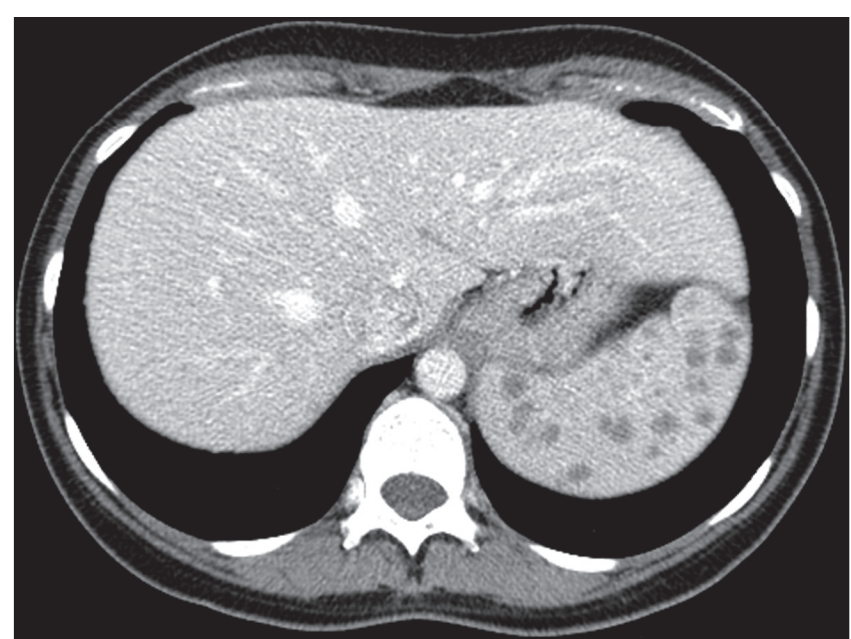

Figure 1) Enhanced computed tomography. Multiple low-attenuation, nonenhancing splenic lesions
The diagnosis of sarcoidosis is based on characteristic imaging findings with compatible clinical features and, if needed, a biopsy showing noncaseating granulomas, thus ruling out other etiologies (2). The clinical triad of bilateral hilar lymphadenopathy, erythema nodosum and arthralgia (Löfgren's syndrome) is classic and considered nearly diagnostic of sarcoid.

The pulmonary parenchyma and the mediastinal and hilar nodal groups are the most commonly affected sites, being involved in up to $90 \%$ of patients. However, any organ system can be involved, and dermatological manifestations, extrathoracic lymphadenopathy and ophthalmic complications are common (1).
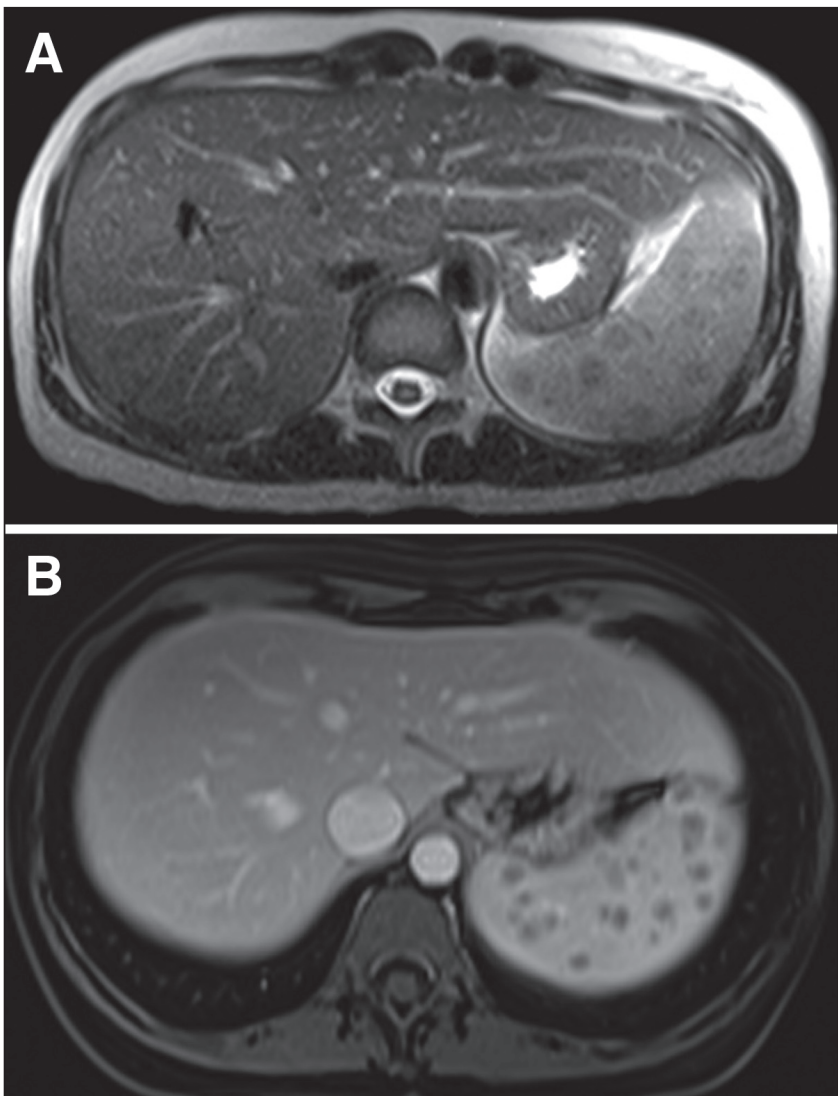

Figure 2) Magnetic resonance imaging of the abdomen. A Axial halfFourier acquisition single-shot turbo spin-echo (HASTE) T2: multiple T2 hypointense splenic lesions. B Axial volumetric interpolated breath-hold examination (VIBE) with FS postcontrast portal venous phase: multiple nonenhancing splenic lesions

${ }^{1}$ Section of Body Imaging, Department of Diagnostic Imaging; ${ }^{2}$ Division of Gastroenterology, Department of Medicine, University of Calgary, Calgary, Alberta

Correspondence: Dr Paul Beck, Division of Gastroenterology, University of Calgary, 3330 Hospital Drive Northwest, Calgary, Alberta T2N 4N1.

Telephone 403-220-4500, fax 403-270-0995, e-mail plbeck@ucalgary.ca

Received for publication June 28, 2011. Accepted July 3, 2011 


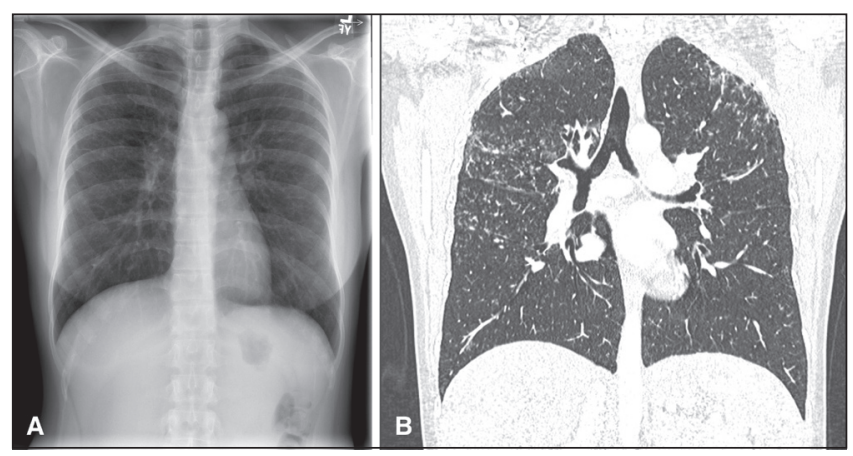

Figure 3) Chest radiograph. A Diffuse nodular interstitial process. B Coronal computed tomography of the chest: diffuse nodular-interstitial pattern in a perihilar distribution with an upper lobe predominance

The abdominal viscera can also be involved with sarcoid, with the liver being the most frequently involved organ. Splenic involvement is reported in up to $40 \%$ to $60 \%$ of patients (3). Splenic involvement with sarcoid usually presents as either splenomegaly or, less commonly, as multiple focal splenic lesions, and patients are often asymptomatic or can present with systemic constitutional symptoms.

Laboratory findings such as disturbances of calcium metabolism are common with sarcoidosis and include hypercalcuria, hypercalcemia and elevated serum angiotensin-converting enzyme levels $(2,4)$.

Two-thirds of patients with sarcoidosis will go into remission, while the remaining one-third of patients can experience unrelenting disease. Fewer than $5 \%$ of patients die from sarcoid, usually from pulmonary fibrosis with respiratory failure or, less commonly, from cardiac or neurological involvement (2). Treatment of sarcoidosis usually begins with corticosteroids, with reservation of immunosuppressive therapy for aggressive or recurrent disease (1).

Multiple focal splenic lesions can be found in $6 \%$ to $33 \%$ of patients with sarcoidosis, with most of the lesions being small (mean diameter of $1.0 \mathrm{~cm}$ ) and up to $50 \%$ of these patients having associated hepatic lesions (3).

Dynamic gadolinium-enhanced MRI is useful in further characterizing splenic lesions and narrowing the differential diagnostic considerations. Classically, sarcoidosis-related splenic lesions are noted to be T1 and T2 hypointense, and are found to be relatively hypoenhancing (Figures 2A and 2B). Infectious lesions (tuberculosis and histoplasmosis) tend to be less well defined and moderately T2 hyperintense, demonstrating more peripheral low-grade enhancement. Benign lesions such as

\section{REFERENCES}

1. Statement on sarcoidosis. Joint Statement of the American

Thoracic Society (ATS), the European Respiratory Society (ERS) and the World Association of Sarcoidosis and Other

Granulomatous Disorders (WASOG) adopted by the ATS Board of Directors and by the ERS Executive Committee, February 1999 Am J Respir Crit Care Med 1999;160:736-55.

2. Iannuzzi MC, Rybicki BA, Teirstein AS. Sarcoidosis. N Engl J Med 2007;357:2153-65.

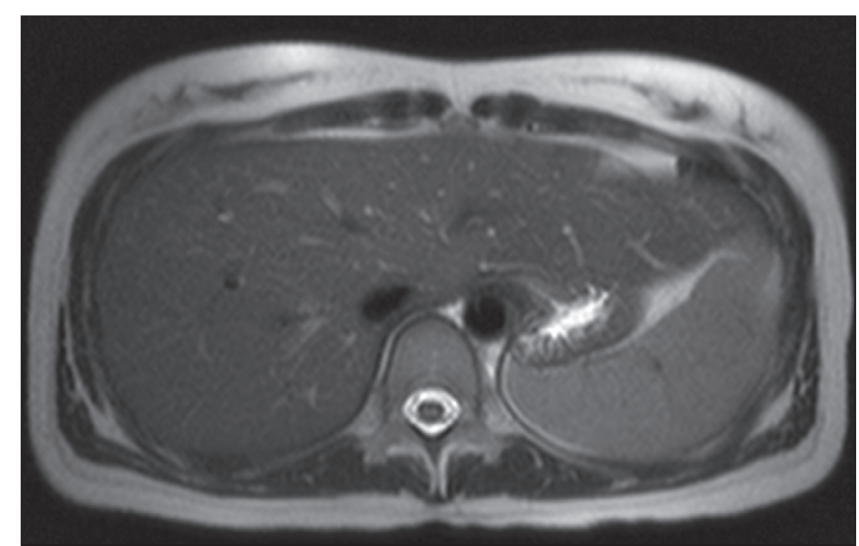

Figure 4) One-year follow-up magnetic resonance image of the abdomen using axial T2 half-Fourier acquisition single-shot turbo spin-echo (HASTE): decrease in the size and number of multiple T2 hypointense splenic lesions

hemangiomas and hamartomas are usually well defined and T2 hyperintense, and demonstrate significant enhancement (hemangiomas early and hamartomas later). Malignant lesions, such as lymphoma, are classically $\mathrm{T} 1$ and T2 isointense or occasionally hypointense, and are relatively hypovascular but can become isoenhancing on delayed imaging. Metastatic lesions are rarely isointense or hypointense on T2 imaging, usually demonstrating low-grade T2 signal and peripheral enhancement (5).

In a study of symptomatic patients with multiple $(>5)$ splenic lesions (3), the most probable etiologies were found to be lymphoma, infection and sarcoid, while in asymptomatic patients, the most likely etiologies were nonlymphomatous metastatic diseases, benign tumours (such as hemangiomas and hamartomas) and sarcoid.

\section{SUMMARY}

MRI is helpful in characterizing splenic lesions, but there can be significant variability and overlap in the appearance of both benign and malignant splenic lesions; thus, a confident diagnosis can be difficult to make. Knowledge of splenic sarcoidosis and its clinical presentation and classic imaging appearance, and further assessment for any other associated findings suggestive of systemic sarcoidosis is critical to avoid missed or delayed diagnosis - specifically, to avoid over-diagnosis of malignant disease because multiple splenic lesions can be the initial presentation of sarcoidosis.

3. Warshauer DM, Lee JK. Imaging manifestations of abdominal sarcoidosis. AJR Am J Roentgenol 2004;182:15-28.

4. Elsayes KM, Narra VR, Mukundan G, Lewis JS Jr, Menias CO, Heiken JP. MR imaging of the spleen: Spectrum of abnormalities. Radiographics 2005;25:967-82.

5. Warhauser DM. Splenic sarcoidosis. Semin Ultrasound CT MR. 2007;28:21-7.

The Canadian Journal of Gastroenterology is now considering a limited number of submissions for IMAGE OF THE MONTH. These will be based on endoscopic, histological, radiological and/or patient images, which must be anonymous with no identifying features visible. The patient must consent to publication and the consent must be submitted with the manuscript. All manuscripts should be practical and relevant to clinical practice, and not simply a case report of an esoteric condition. The text should be brief, structured as CASE PRESENTATION and DISCUSSION, and not more than 700 words in length. A maximum of three to four images can be submitted and the number of references should not exceed five. The submission may be edited by our editorial team.

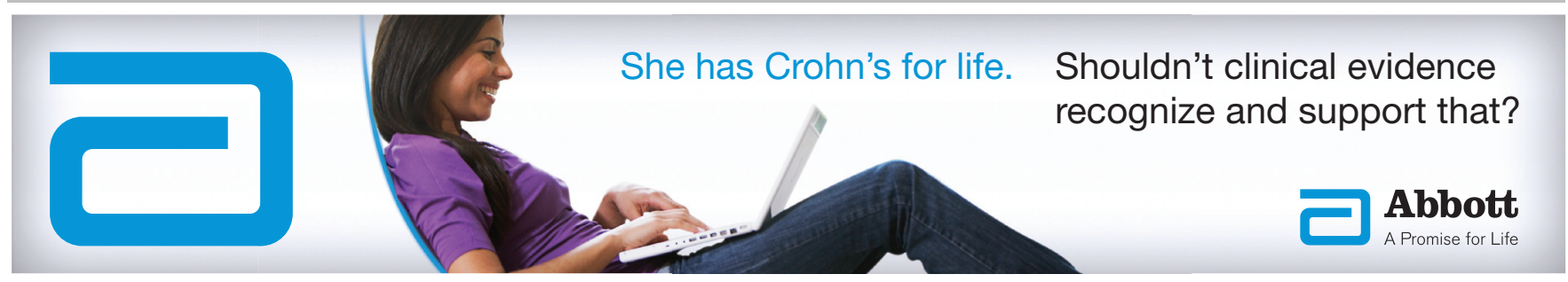




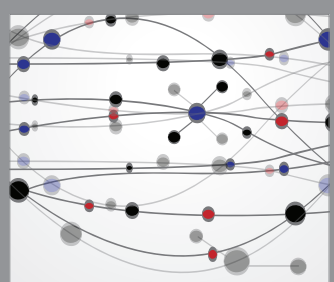

The Scientific World Journal
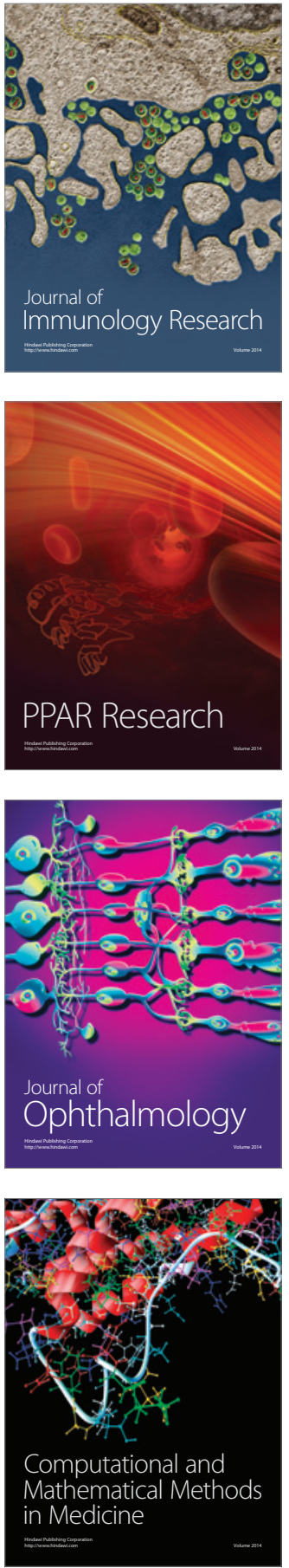

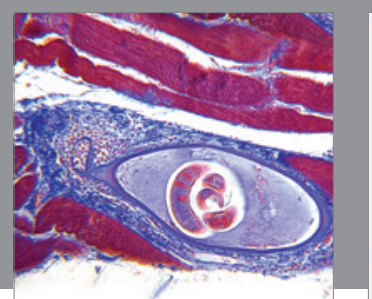

Gastroenterology Research and Practice

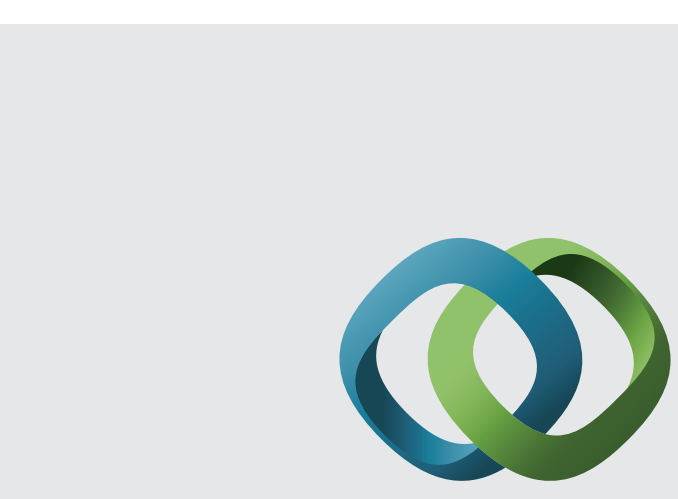

\section{Hindawi}

Submit your manuscripts at

http://www.hindawi.com
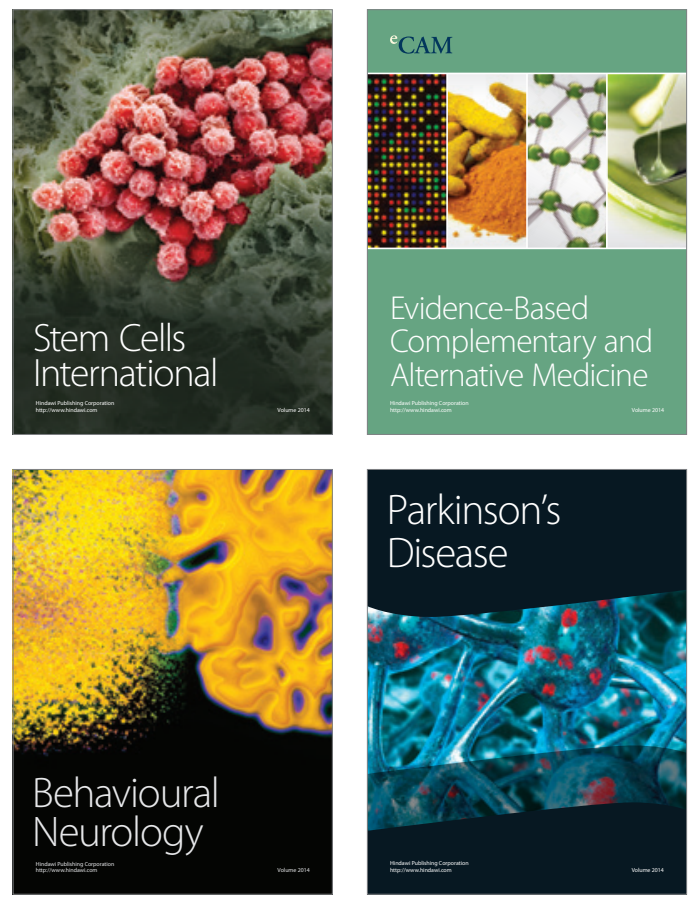
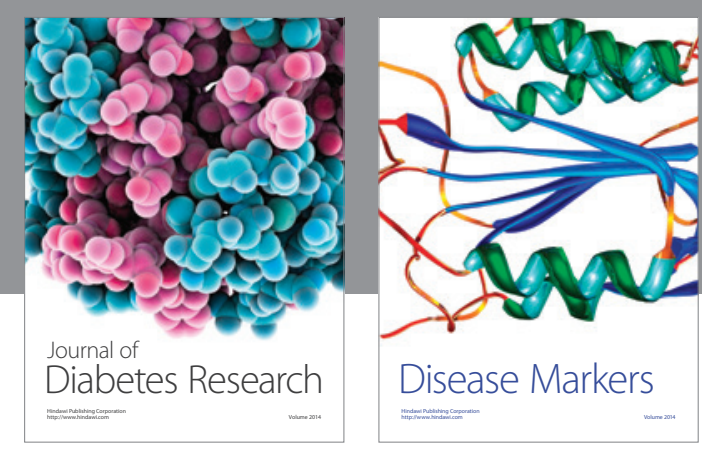

Disease Markers
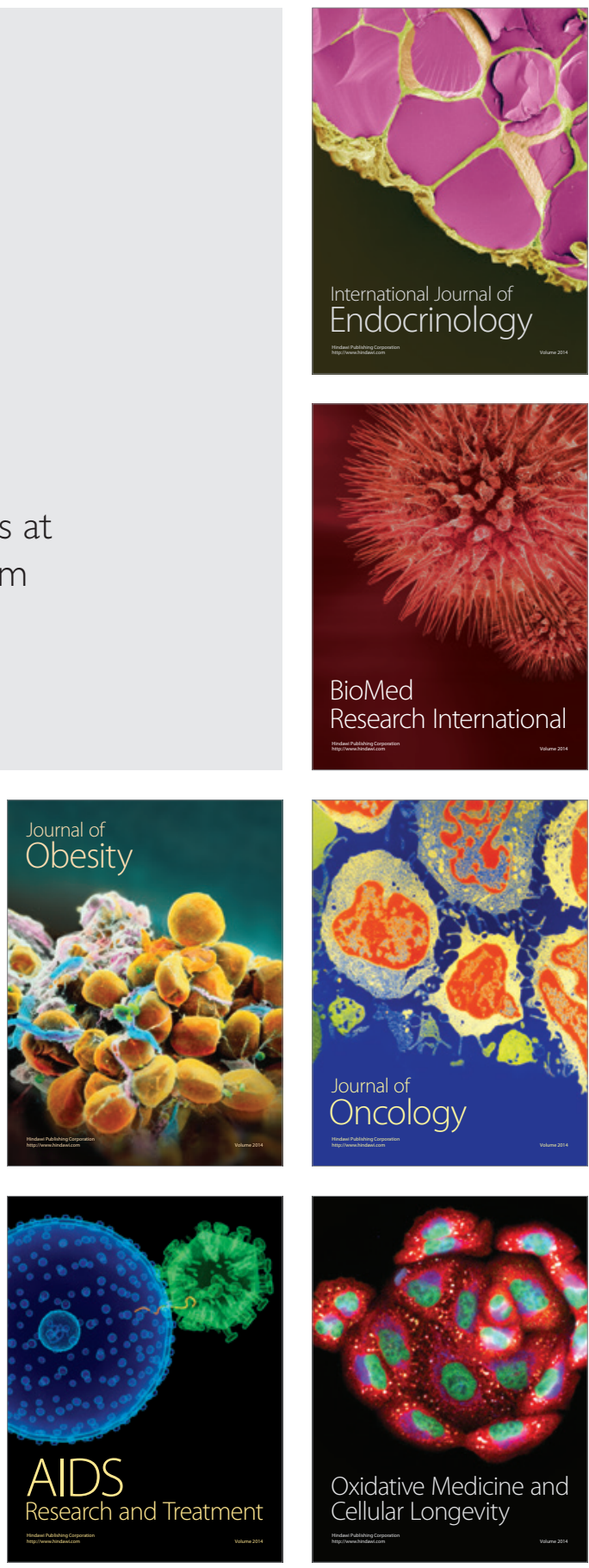